\title{
reviews
}

columns

\section{Computers in Psychiatry}

Fionnbar Lenihan (ed.)

Gaskell, 2006, f22.50, 224 pp. ISBN: 1904671217

My usual reaction when faced with a book of this sort is to snort in irritation and to doubt if it can teach me anything. This uncharacteristic (I like to think) arro gance stems from my many years as a self-taught information technology enthusiast and then, more recently, as a disillusioned Cassandra. However, this book proved me wrong. In spite of attempting to be being all things to all men, it caters well for both for the computer novice and the more experienced user.

It begins with some very basic information about what a computer is made up of, what it does - and even how you connect the various bits together. This may be useful for older colleagues who have somehow managed to avoid any contact with computers; however, anyone who has had a computer for more than 6 months will view it briefly and skip it. The chapters on software again contain basic nuts-and-bolts information about what different kinds of packages actually do. But, for the more experienced user, they have some very useful hints about the more advanced skills that are stil within the grasp of the non-expert. These are the things that - after you've learnt how to string a sentence together on the word processor - you know you ought to get to grips with but somehow never do for me, it was the 'styles' facility, which lets you format all parts of a text document with just a single keystroke, potentially saving huge amounts of time and drudgery. The authors also manage to make clear the differences between spreadsheets and databases, especially pertinent as I see Excel spreadsheets used as surrogate databases most days.

One thing I particularly liked about this book was that it tried, harder in some chapters than in others, to look beyond the monolithic world of Microsoft software. One might view this as wasted effort as the NHS seems to have pretty much standardised on this company's products. However, no one knows what the future holds and the fact is that there is some extremely capable, free software that is the equal, or better, of anything

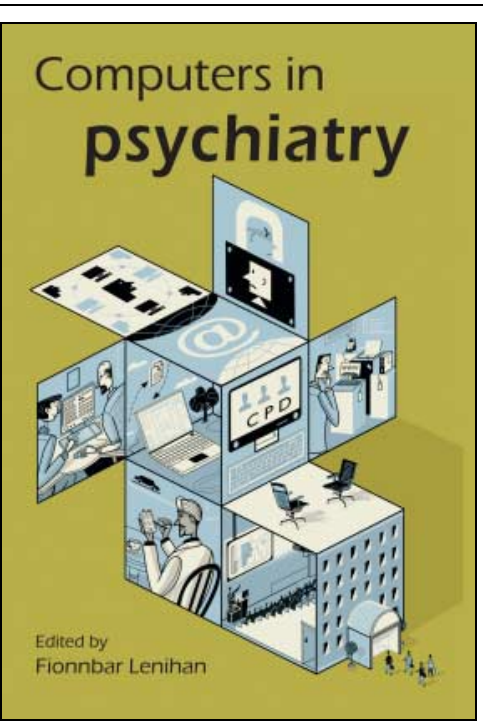

that Microsoft can offer. Much of it is 'open source', which means that the source code is available to anyone who wants it - this means that it is worked on by hundreds or thousands of enthusiasts across the world and so can sometimes be developed far more quickly than commercial software. So, it is not unrealistic to consider the possibility that, in time, this model of software development and provision might become the norm. The fact that it is free means that it is a realistic option for the many parts of the world where our colleagues cannot afford commercially marketed software.

As well as this more global dimension the book has an excellent chapter on the novel legal ramifications generated in the UK by this new technology and a helpful section on computer security. This deals not just with the hardware/software issues but with the tricky 'human technology' of choosing passwords that are hard to guess. This has helped me to fee slightly less irritated with the regular and rather finicky demands I receive for password changes and with the numbers of letters and numbers they contain. Some of this will be familiar to anyone who has ever opened a computer magazine, for example 'never reply to spam email'. Some is, again of the 'known about but never tried' variety such as the use of digital signatures for emails.

Overall this is a modestly priced and well written book that will help beginners to find their feet and more skilled users to broaden their view of what this still complex technology can do for them.
Philip Timms Consultant Psychiatrist, South London and Maudsley NHS Trust, London, email: philip.timms@slam.nhs.uk

doi: $10.1192 / p b . b p .106 .011122$

\section{Good Practice in Adult Mental Health}

Tony Ryan \& Jacki Pritchard (eds) Jessica Kingsley, 2004, f18.95, pb, 352 pp. ISBN 184310217X

Did I find this book interesting? Yes, but would I recommend it to everyone? No. In most respects it is a fascinating collection of individual viewpoints and opinion, many of which may be widely shared, but I am not quite sure what it really intends to be. On the one hand it seems to set out to be an introductory book on adult mental health, a primer perhaps, but the title suggests something more and the editors do say it is designed for the reader to 'dip in and out of sections', which rather implies a reference function.

I don't really think it works as an introductory text or reference. It is not really suitable for those new to mental health as there is much missing and the construction and order of the chapters appears rather haphazard. There is too much opinion for a reference work and some things are stated as fact with which I would take issue. The editors do point out that they have not been able to be comprehensive, but certain things are too obvious by their absence. For example, there is no mention anywhere of psychodynamics and, although there is a chapter on 'medication management' there is no proper explanation of the rationale behind the use of psychotropic drugs.

However, much of the book is interesting and thought-provoking, and one or two of the chapters (such as 'Personal experiences of mental health and illness') are utterly absorbing. The case studies and some of the good practice points are also a nice touch.

So, although I do not think it is essential reading for anyone, having a copy to hand to dip into from time to time might help stimulate thought and provoke debate.

Hugh Griffiths Deputy National Director for Mental Health, Department of Health Whitehall, 79 Whitehall, London SW1A 2NL, email: hugh.griffiths@dh.gsi.gov.uk

doi: 10.1192/pb.bp.105.006536 